\title{
Adaptive Traffic-Signal Control using Discrete Event Simulation Model
}

\author{
Ahmad Aljaafreh \\ Communications and Computer Engineering Department \\ Tafila Technical University \\ Tafila, Jordan
}

\author{
Naeem Al-Oudat \\ Communications and Computer Engineering Department \\ Tafila Technical University \\ Tafila, Jordan
}

\author{
Ma'en Saleh \\ Communications and Computer Engineering Department \\ Tafila Technical University \\ Tafila, Jordan
}

\begin{abstract}
Different factors affect the process of choosing the appropriate traffic signal controller to solve the traffic conflict on an intersection. Important factors are; number of phases and vehicles arrival rates. Sequence of phases, timings of traffic signals and length of cycle are the most important parameters that all traffic signal controllers aim to optimize one or more of them. One of the major performance measures of traffic signal controller is the average waiting time of vehicles. To compare different kinds of traffic signal controllers, a discrete event simulation model of traffic signal controller on a single intersection is developed using Matlab/Simulink/Simevents. In this paper, three algorithms are proposed to reduce the average waiting time at intersections. The proposed algorithms are compared to the base-line fixed-time controller through extensive simulation experiments. All the proposed algorithms outperforms the base-line algorithm when there is a high variance on the traffic flow. One of the proposed algorithms that adapts both green intervals and cycle length, $A W$ Variable $C$, outperforms other algorithms, including base-line, under all conditions, but this is on the expense of more computational overhead and more input parameters.
\end{abstract}

\section{Keywords:}

Traffic-control, Simulation, Matlab/Simulink, Fixed green-interval controller, Adaptive green-interval algorithm

\section{INTRODUCTION}

Managing and controlling the traffic is becoming a serious problem especially with a large growing number of automobiles and marginal increase in capacities of the roads around the globe. One of the bottle necks in face of achieving an efficient and smooth flow of traffic is intersection traffic light signal control. Several parameters are controlling how efficient a traffic light signal is. Arrival rates, number of junction's entries and number of lanes in each entry are the most important factors on traffic light signal management. The main purpose of any management strategy of traffic light signal is minimizing the average waiting time of the vehicles on junctions. This goal can be achieved, with differences in performance, using different kind of traffic light controller approaches.

Traffic-light controllers can be categorized to two types; the conventional fixed-time controller and the real-time adaptive controller. The fixed-time controller uses a preset cycle time based on a prior knowledge of traffic flow. The cycle time is the time of one complete rotation including green intervals plus the change and clearance intervals through all phases. The advantage of fixed time control is the simplicity of the control where the disadvantage is that it does not adapt to variable traffic situations. Adaptive controller uses sensors input to activate a change in the cycle time and/or the phase sequence[1]. The system detects vehicles flow and queue length using electronic sensors embedded in the pavement or through images as in [1]. A fuzzy controller is proposed in [2] to dynamically control the traffic light timings and phase sequence based on traffic density and delay on each approach on a single intersection. Two functions based on if-rules are used to determine the next phase and weather to extend the green time of the current phase or not. Simulation results using Matlab/Simulink software show that the performance of fuzzy controller is better than fixed controller in heavy traffic conditions. A comparison between fuzzy logic controller and fixed-time controller is done in [3]. Simulation studies show that the fuzzy logic controller has better performance. An improved discrete event simulation model of traffic light control using Matlab/Simulink/SimEvents on a single intersection is described and validated in [4]. Each intersection stream is modeled as an $\mathrm{M} / \mathrm{M} / 1$ queue. Time between vehicle arrivals is modeled using exponential distribution. The number of vehicles and the average waiting time in each stream are used as the performance indexes for traffic light signal control. An adaptive time control algorithm is developed in [5] to compute the red/green light duration for each traffic signal found by using the conflict directions matrix. Simulation of fuzzy traffic controller for controlling traffic flow at multi-lane isolated signalized intersection is presented in [6]. An intersection model consists of two lanes with different values of vehicles queue length and waiting time in each approach, was proposed. The maximum values of vehicles queue 
length and waiting times are selected as the inputs to the fuzzy controller.

In [8], authors proposed a solution to a single intersection congestion problem based on second type fuzzy logic controller. Authors in [9] provide an optimization method for the traffic control at an isolated intersection with the aim of minimizing traffic emissions. Wanjing Ma et al. in [10] formulated the traffic light control problem as a binary mixed integer linear program to maximize the person capacity of the intersection and solved this program using a branch-and-bound method. In [11], authors provided an extensive survey of computational intelligence used in controlling traffic light signals.

The main goal of this research is prevention of traffic jam and reduction of driver waiting time. This could be achieved by optimizing the functioning of traffic signal. Traffic signal optimizing is considered one of the most effective measures to address traffic congestion [12]. However it is complex and blind due to randomness and abnormal situations [12]. In a previous work by Ahmad Aljaafreh and Naeem Al Oudat [13], some scheduling algorithms were tried to solve congestion problem at intersections. In this research, a discrete event simulation model is developed, verified and validated. The model is tested in different scenarios. Thus, the objective of this paper is to determine the optimized timing parameters for traffic signal by simulation. Designing a real-time proactive adaptive control model for traffic will enhance the performance of traffic light.

The rest of this paper is organized as follows. Section 2 details the optimization parameters used in this paper. Fixed and adaptive algorithms are discussed in Section 3 Simulation studies using Matlab/Simevent are presented along with some results in Section 4 Finally paper is concluded and some future work directions are given in Section 5

\section{OPTIMIZATION PARAMETERS}

Several parameters (dimensions) are usually considered altogether one or two at a time for optimization at an intersection to minimize the average waiting time, emissions, number of stops, etc. These parameters are shown in Figure 1 Green interval is the time an

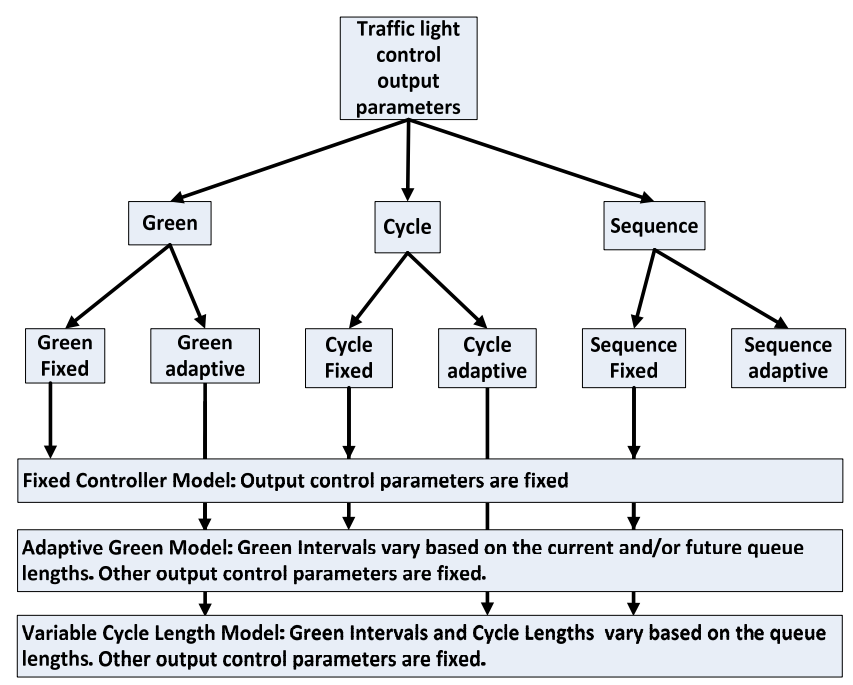

Fig. 1. Intersection optimized parameters. intersection entry is open for vehicles to pass. Cycle is the sum of green intervals for all entries and yellow light intervals between them, see Figure 2 And phase sequence is how different entries take turns in opening (green light is on).

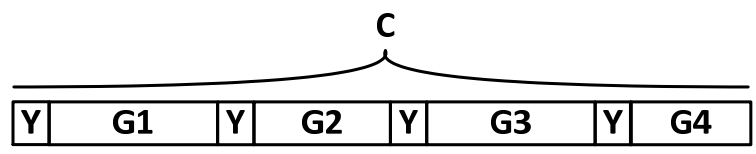

Fig. 2. Green intervals and yellow times in a cycle of four entries

\section{ADAPTIVE ALGORITHMS}

A Simulink based models are created and described in this section using Simevents block set. An adaptive controller that is based on the green interval and/or cycle length is described along with the baseline model of fixed cycle, green interval and sequence. Three output control parameters are of great importance in controlling traffic light signals. These controlled parameters are; cycle length, green light intervals, and sequence of intersection entries (Figure 1] shows these parameters)

\subsection{Fixed Green-Interval Model}

A Simulink model for fixed green interval is built as in Figure 3 In this model an optimal cycle length is determined using an extensive simulation runs. An intersection of four entries is used. All entries have the same arrival rate of traffic. Arrival rate variates are drawn from an exponential distribution. A yellow light period is taken as a transition barrier between subsequent green intervals. Then the green interval, $G_{I N T}$ is:

$$
G_{I N T}=\frac{\text { CycleLngth }-4 * \text { YellowTime }}{4}
$$

where YellowTime is the switching time between red and green lights.

The optimal cycle length is the one that gives the minimum average waiting time for a specific arrival rates. After running the Simulink model for different arrival rates, we get the optimal cycle lengths as shown in Figure 4. Fixed green interval controller is called $A W$ Fixed. Figure 4 shows that when the arrival rate increases the required cycle length, which optimally services the vehicles at the junction entries, increases. This behavior is due to the fact that the number of queued vehicles at the junction increases and hence a larger green interval is required to clear as many as possible vehicles at any given entry. Yellow time can be considered as a waste-of-time needed to switch between different lanes. This waste-of-time is fixed for each cycle regardless of the cycle time. Thus, cycle time should be determined carefully (Cycle time calculation is directly connected to the queue lengths and service times of vehicles on each entry).

\subsection{Adaptive Green-Interval Model}

The algorithm proposed here, depends on varying the green interval based on the queue length for each entry at the intersection. As the arrival rate increases at an entry the queue length increases at the same entry. Figure 1 shows the proposed model in relation to the three controlled parameters named as second model. The core of the model in Figure 5 is the adaptive green-interval calculation algorithm, $A W$ Adaptive. Algorithm 1 shows the algorithm of $A W$ Adaptive. Intuitively there is a waste of time to open an entry (green 


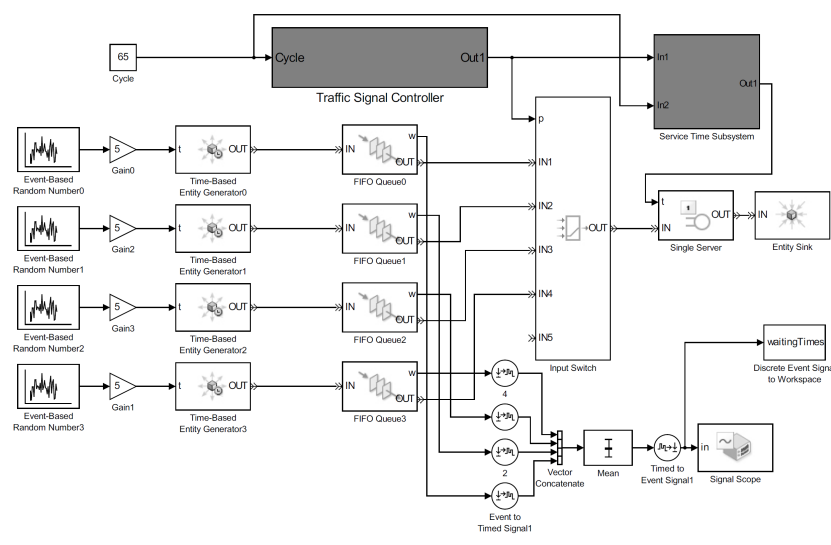

Fig. 3. Simulink model for fixed parameters traffic light controller.

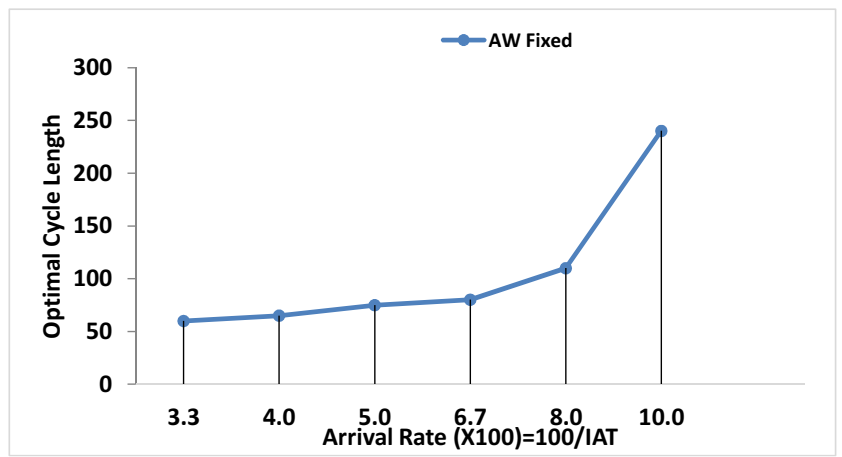

Fig. 4. Optimal cycle length vs. inter arrival time (1/arrival rate) of vehicles.

interval) with a small queue length for an interval that is equal to the one with a greater queue length. Based on that, an entry is opened for an interval of length proportional to its queue length, as can be seen in the algorithm.

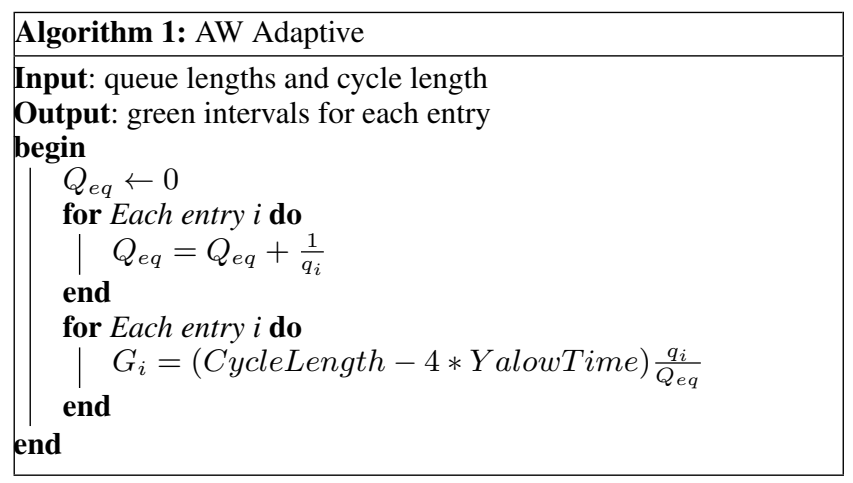

\subsection{Adaptive Green-Interval Algorithm Based on Prediction}

The same model in Figure 5 is used while the only change is plugging the control algorithm (Algorithm 2) into its core. A sequence of phases, assuming only four, is shown in Figure 2

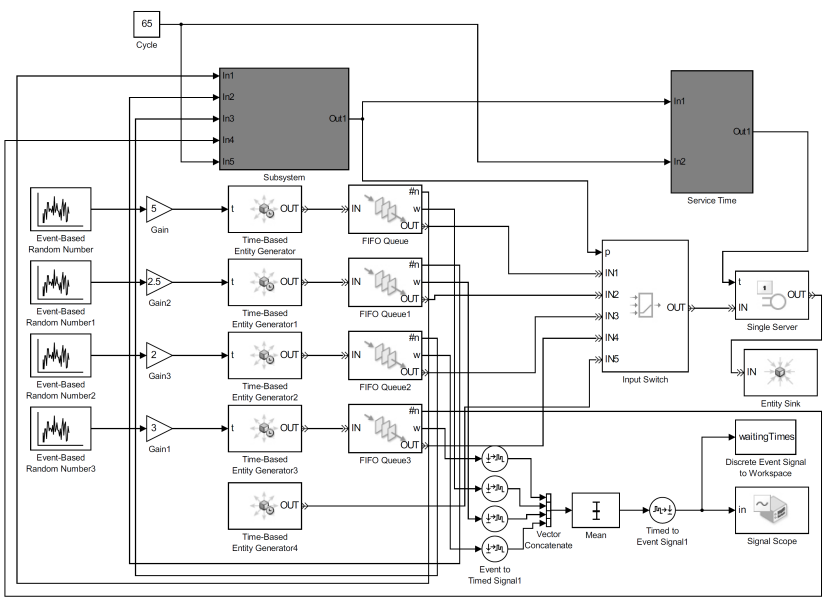

Fig. 5. Simulink model for adaptive traffic light controller. In the previous algorithm green intervals for the phases are determined based on queue lengths at the beginning of the cycle, $C$. This method favor the first phase over other phases where their queues will most likely be longer and therefore they do not get a fair green slot that is proportional to their queue lengths. Although future queue lengths are not known, they can be predicted based on the arrival rates, $A r_{i}$, and present queue lengths, $q_{i}$. From Figure 2 . as an example, green intervals, $G_{i}$, can be calculated as:

$$
\begin{array}{r}
G_{1}=\frac{q_{1}}{q_{t}} C \\
G_{2}=\frac{q_{2}+G_{1} A r_{2}}{q_{t}} C \\
G_{3}=\frac{q_{3}+G_{1} A r_{3}+G_{2} A r_{3}}{q_{t}} C \\
G_{4}=\frac{q_{4}+G_{1} A r_{4}+G_{2} A r_{4}+G_{3} A r_{4}}{q_{t}} C
\end{array}
$$

where; $q_{t}=q_{o r g}+q_{a c c}^{G}+q_{a c c}^{Y}$ is the total queue lengths that is the sum of known queue lengths at the beginning of the cycle, $q_{\text {org }}=$ $q_{1}+q_{2}+q_{3}+q_{4}$, and accumulated queue lengths during green intervals, $q_{a c c}^{G}=G_{1}\left(A r_{2}+A r_{3}+A r_{4}\right)+G_{2}\left(A r_{3}+A r_{4}\right)+G_{3} A r_{4}$ and yellow intervals, $q_{a c c}^{Y}=Y\left(A r_{2}+2 A r_{3}+3 A r_{4}\right)$.

In the above, $G_{1}, G_{2}, G_{3}$ and $G_{4}$ are not known and the given equations are not enough to calculate them. However a bound on the green sizes can be given. Notice that any green size, $G_{i}$, lies in the interval $\left[G_{i}^{M I N}, G_{i}^{M A X}\right] . G_{i}^{M I N}$ and $G_{i}^{M A X}$ are given as follows:

$$
\begin{gathered}
G_{i}^{M A X}=\frac{q_{i}}{\sum_{j}^{N} q_{j}} C \\
G_{i}^{M I N}=\frac{q_{i}+\sum_{w=1}^{i-1} G_{w} A r_{i}+Y \sum_{b=2}^{i} A r_{i}}{\sum_{j}^{N} q_{j}+Q_{g}+Q_{y}} C
\end{gathered}
$$

where;

$$
\begin{gathered}
Q_{g}=\sum_{w=2}^{N}\left(G_{w}^{M A X} \sum_{(y=w+1)}^{N} A r_{y}\right) \\
Q_{y}=Y \sum_{b=2}^{N}(b-1) A r_{b}
\end{gathered}
$$


A reasonable choice of $G_{i}$ is taking it as the mid-way between $G_{i}^{M I N}$ and $G_{i}^{M A X}$ starting from green interval number one, $G_{1}$ :

$$
G_{i}=\frac{G_{i}^{M A X}+G_{i}^{M I N}}{2} C
$$

In calculating $G_{i}^{M I N}$ the previous G's calculated as above are substituted in the equation. Sum of green intervals thus calculated, should fit in the cycle. Any difference should be compensated by distributing it on all green intervals. Algorithm 2 shows this procedure.

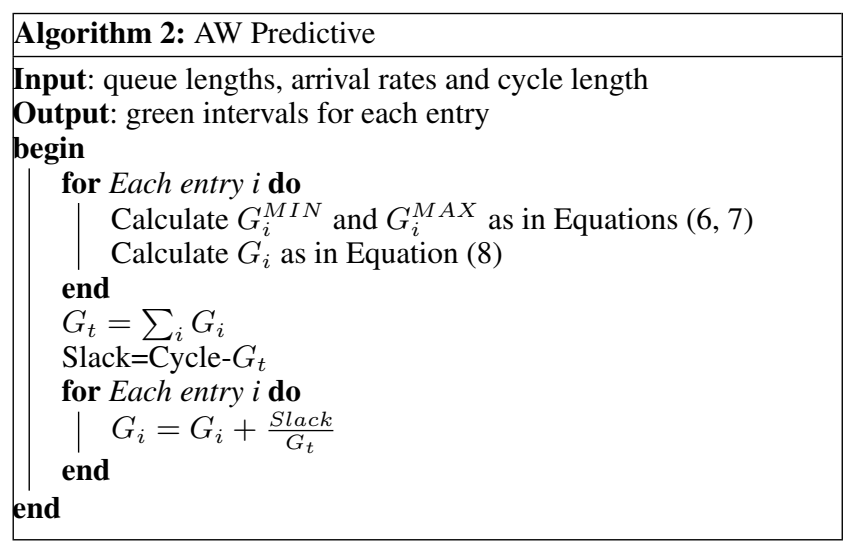

\subsection{Adaptive Green- and Cycle-Lengths Algorithm}

The same model in Figure 5 is used while the only change is plugging the control algorithm (Algorithm 3 into its core. In this algorithm, service times are used to calculate the time needed to empty the queue at an entry. The service time model used in this paper is given in Equation 9 .

$$
\begin{array}{r}
S_{i}=S_{(i-1)}+4-0.3 * i, 1 \leq i<8, S_{0}=4 \\
S_{i}=S_{(i-1)}+2,8 \leq i
\end{array}
$$

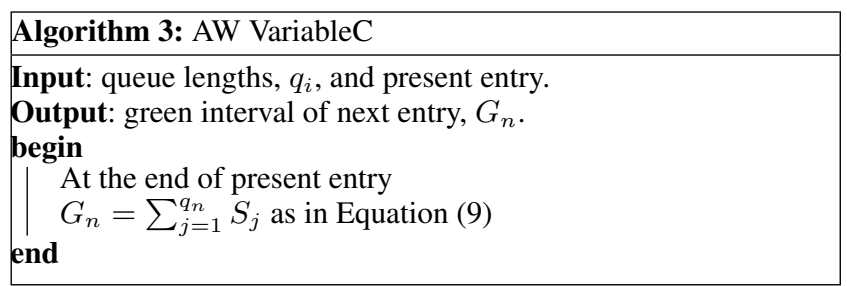

Algorithm 3 shows the procedure called when its current entry turn to open. Based on the service time model and on the queue length at that moment, the green interval for this entry is calculated. Since the queue lengths are not a fixed parameters and they are varying all the time, the cycle is also variable.

\section{SIMULATION STUDIES}

The proposed algorithms were assessed using extensive simulation experiments. 51 runs using Independent and identically distributed (IID) random variables for 2000 simulation time units were conducted. Then the average of the performance parameter, i.e., waiting times, values was calculated. Note that YellowTime parameter is set to 4 seconds.

Results of the simulation study are presented and discussed in this section. Two interesting results are shown. The results are a comparison of the performance of the proposed models and $A W$ Fixed algorithm.

\subsection{Impact of Equal Arrival Rates}

In this experiment we assumed equal arrival rates at all entries. Figure 6 shows the average waiting time vs. arrival rates for the proposed algorithms (AW Adaptive, AW Predictive and $A W$ Variable $C$ ) and baseline algorithm (AW Fixed). As expected baseline algorithm outperforms $A W$ Adaptive and $A W$ Predictive, since the AW Fixed algorithm in this case gives the optimal lower bound on average waiting time. While $A W$ VariableC outperforms all other algorithms, due to the high adaptability for variations in arrival rates and using just the required cycle length without wasting much time during which the traffic light signal could be idle. This ability comes with a price, where the controller looks at the queue lengths and arrival rates at the end of each green interval to calculate the length of the next one, while this kind of parameters reading and calculations is done only at the end of the cycle in other algorithms. Figure 8 shows cycle lengths of $A W$ Variable $C$ as compared to other algorithms where there cycle lengths are determined in advance.

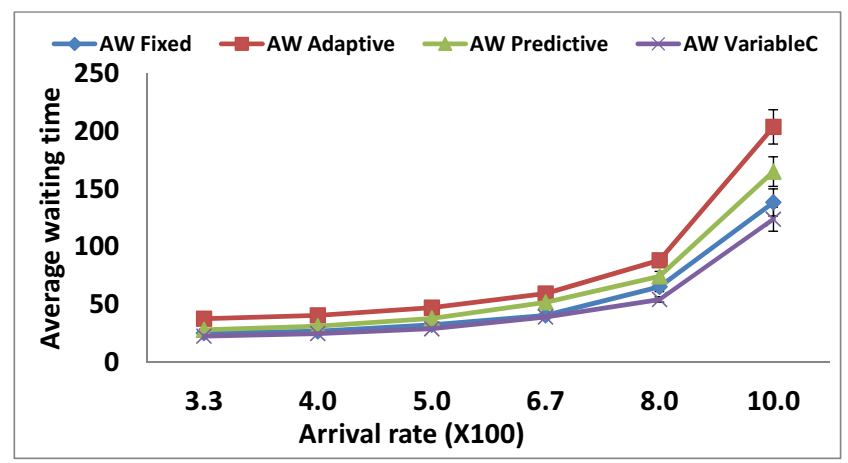

Fig. 6. Average waiting time vs. arrival rates for the proposed algorithm and baseline algorithm.

\subsection{Impact of Variance in Arrival-Rate Increase}

In this experiment, the cycle is fixed on 65 seconds, except for $A W$ Variable $C$ algorithm, and the arrival rates for the entries are varied. The variations in arrival rates are increased above the optimal arrival rate of $1 / 25$. Figure 7 shows the average arrival rates vs. variance of rate increase for the proposed algorithms and the baseline algorithm.

Baseline algorithm outperforms $A W$ Adaptive and $A W$ Predictive algorithms when the variance is low. As the variance increases, the proposed algorithms outperform the baseline algorithm. Furthermore, the performance of the proposed algorithm keeps 
almost a constant average waiting time for all values of the variance. Note that $A W$ Variable $C$ algorithm outperforms all other algorithms due to its high adaptability for the variations in input parameters.

Figure 9 shows the variations in cycle lengths for $A W$ Variable $C$ algorithm under different variances in arrival rate increases. This figure shows the amount of cycle lengths that are wasted for other algorithm without usage for consuming vehicles at the intersection entries. Wasted times in cycle lengths can be easily seen from the difference between the two curves in Figure 9

This result shows the power of the proposed algorithms, which shows a resilient performance regardless of the overloading conditions of the junction due to heavy traffic.

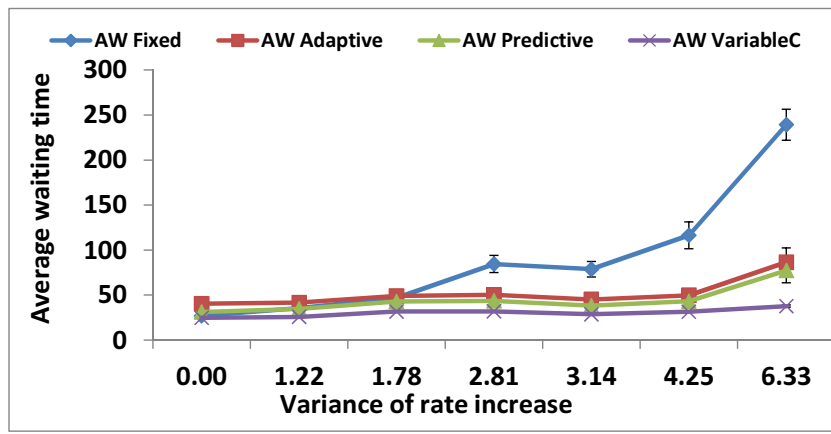

Fig. 7. Average waiting time vs. variance of rate increase for the proposed algorithm and baseline algorithm.

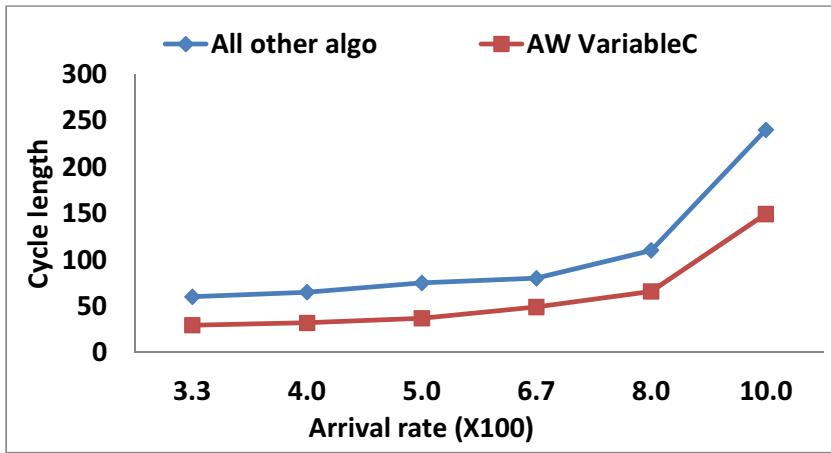

Fig. 8. Cycle length vs. arrival rate for AW VariableC and all other algorithms.

\section{CONCLUSIONS AND FUTURE WORK}

In this paper, three algorithms are proposed and tested through extensive simulations using a discrete event model for traffic light controller. In the way, three control parameters were identified namely; green-interval, cycle and sequence of entry openings. To study the proposed algorithms, two simulation experiments were conducted. In the first one, the proposed algorithms ( $A W$ Adaptive, $A W$ Predictive and $A W$ Variable $C$ ) were compared with fixed green-interval baseline algorithm ( $A W$ Fixed). Results showed that $A W$ VariableC outperformed all other algorithm including baseline algorithm due to its high adaptability to the variations in

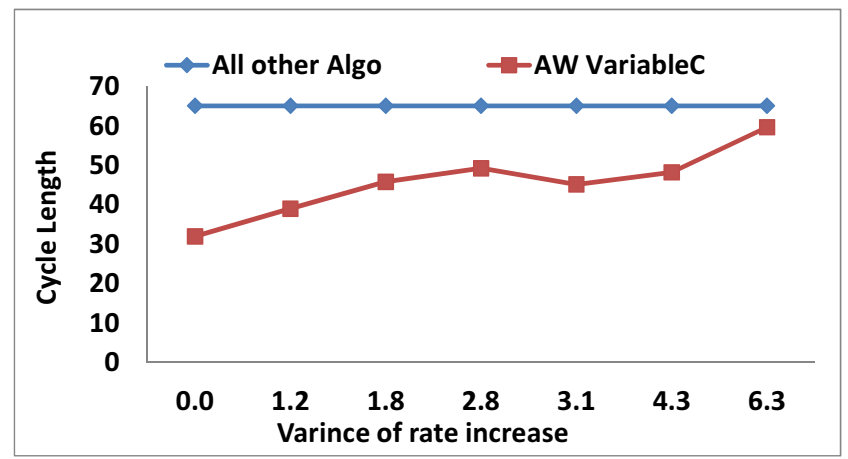

Fig. 9. Cycle length vs. variance of rate increase for AW VariableC and all other algorithms.

input parameters. In the second experiment, the impact of variance in arrival rates for different entries were studied, where results showed that as the variance of arrival rates increased the proposed algorithms outperformed the baseline algorithm.

In this research, adapting green-interval and cycle length were considered. As a future work, the sequence parameter will be considered for adapting. Another future work direction is to consider more than one intersection at a time in a form of distributed control system.

\section{REFERENCES}

[1] George, Anna Merine, FUZZY CONTROLLER FOR AN IMAGE BASED TRAFFIC SYSTEM. International Journal of Management, IT and Engineering, Volume 2, Issue 6, June 2012.

[2] Mwangi, Charles Maina, S. M. Kang'ethe, and G. N. Nyakoe. Design and Simulation of a Fuzzy Logic Traffic Signal Controller for a Signalized Intersection. Scientific Conference Proceedings. 2012.

[3] Center, Artificial Intelligence, and Jalan Semarak. Intelligent traffic lights control by fuzzy logic. Malaysian Journal of Computer Science 9.2 (1996): 29-35.

[4] Sumaryo, Sony, A. Halim, and K. Ramli. Improved discrete event simulation model of traffic light control on a single intersection. QiR (Quality in Research), 2013 International Conference on. IEEE, 2013.

[5] Yousef, Khalil M., Mamal N. Al-Karaki, and Ali M. Shatnawi. Intelligent Traffic Light Flow Control System Using Wireless Sensors Networks. J. Inf. Sci. Eng.26.3 (2010): 753-768.

[6] Soh, Azura Che, Lai Guan Rhung, and Haslina Md Sarkan. Matlab simulation of fuzzy traffic controller for multilane isolated intersection. International Journal on Computer Science and Engineering 2.4 (2010): 924-933.

[7] Bi, Yunrui, et al. Single intersection signal control based on Type-2 fuzzy logic. Computational Intelligence in Vehicles and Transportation Systems (CIVTS), 2013 IEEE Symposium on. IEEE, 2013.

[8] Qian, Rao, et al. A Traffic Emission-saving Signal Timing Model for Urban Isolated Intersections. Procedia-Social and Behavioral Sciences 96 (2013): 2404-2413.

[9] Ma, Wanjing, K. Larry Head, and Yiheng Feng. Integrated optimization of transit priority operation at 
isolated intersections: A person-capacity-based approach. Transportation Research Part C: Emerging Technologies 40 (2014): 49-62.

[10] Zhao, Dongbin, Yujie Dai, and Zhen Zhang. Computational intelligence in urban traffic signal control: A survey. Systems, Man, and Cybernetics, Part C: Applications and Reviews, IEEE Transactions on 42.4 (2012): 485-494.

[11] http://www.mathworks.com/products/matlab/, last accessed
7-4-2014.

[12] Gershenson, Carlos. Self-organizing traffic lights. arXiv preprint nlin/0411066(2004).

[13] Ahmad Aljaafreh and Naeem Al Oudat. Optimized Timing Parameters for Real-Time Adaptive Traffic Signal Controller.

In Proceedings of the 2014 UKSim-AMSS 16th International Conference on Computer Modelling and Simulation (UKSIM '14). 